\title{
LETTER
}

Acute lymphoblastic leukemia

\section{HLA-matched and HLA-haploidentical allogeneic CD19-directed chimeric antigen receptor T-cell infusions are feasible in relapsed or refractory B-cell acute lymphoblastic leukemia before hematopoietic stem cell transplantation}

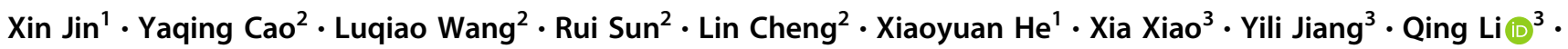 \\ Huan Zhang ${ }^{3} \cdot$ Wenyi $\mathrm{Lu}^{3} \cdot{\text { Cuicui } \mathrm{Lyu}^{3} \cdot \text { Yanyu Jiang }}^{3} \cdot$ Juanxia Meng ${ }^{3} \cdot$ Mingfeng Zhao ${ }^{1,3}$
}

Received: 15 May 2019 / Revised: 16 August 2019 / Accepted: 21 August 2019 / Published online: 18 October 2019

(c) The Author(s) 2019. This article is published with open access

\section{To the Editor:}

Chimeric antigen receptor-modified T (CAR-T) cells exhibit very effective function in elimination of relapsed/refractory B-cell acute lymphoblastic leukemia (R/R B-ALL) [1]. In patients with a high tumor burden or who have undergone heavy treatment, it is not always possible to manufacture an effective therapeutic product $[2,3]$. Here, we describe the first-in-human use of HLA-matched allogeneic CAR-T cells (MCART19 cells) before allogeneic hematopoietic stem cell transplantation (allo-HSCT) and expand the description of HLA-haploidentical allogeneic CAR-T cell (HCART19 cell) infusion reported in previous sporadic cases.

Autologous CAR-T cells are difficult to produce due to high tumor burden or severe treatment. Eight patients with R/R B-ALL who had never received allo-HSCT received allogeneic CAR-T (allo-CART19) cell infusions. As of March 24, 2018, the median follow-up period was 10 months. All patients received more than four treatments before enrollment. One patient was $\mathrm{PH}$ positive (bcr-abl $190+$ ), and seven were PH negative. Three patients had

These authors contributed equally: Xin Jin, Yaqing Cao, Luqiao Wang

Supplementary information The online version of this article (https:// doi.org/10.1038/s41375-019-0610-x) contains supplementary material, which is available to authorized users.

Mingfeng Zhao

mingfengzhao@sina.com

School of Medicine, Nankai University, Tianjin 300071, China

2 The First Central Clinical College, Tianjin Medical University, Tianjin 300070, China

3 Department of Hematology, Tianjin First Central Hospital, Tianjin 300192, China mutations associated with poor prognosis. All patients received lymphodepleting chemotherapy with fludarabine/ cyclophosphamide. Except for patient 4, all patients exhibited a proportion of blast cells in the bone marrow that exceeded $80 \%$. A median of $51.5 \%$ (range, 30-81\%) of the infused T-cell mixture was CAR-T cells expressing antiCD19. The patients numbered $1-4$ received MCART19 cell infusions, and the other four patients received HCART19 cell infusions. The median infusion of allo-CART19 cells in the patients was $2 \times 10^{6} / \mathrm{kg}$ (range, $5 \times 10^{5}-1 \times 10^{7}$ ). Because infusion-related toxicity was mild, the four patients infused with the HCART19 cells (up to $1 \times 10^{7} / \mathrm{kg}$ ) received a higher dose than those infused with the MCART19 cells. All details are summarized in Tables 1 and S1.

All patients developed toxicity and required drug intervention after allo-CART19 cell infusion (Tables 1 and S2). Neurotoxicity and grade $\geq 3$ cytokine release syndrome (CRS) occurred in three patients (patients 1,2, and 4) treated with the MCART19 cells, and these patients developed hyperthermia, chills, muscle aches, apathy, loss of consciousness and cognitive disturbance accompanied by high levels of inflammatory cytokines and MCART19 cells in the peripheral blood (Figs. S1A-F and 1a). Under methylprednisolone treatment, anti-IL-6 antibody (tocilizumab) treatment, and other supportive treatments, patients 1 and 4 gradually recovered, but patient 2 deteriorated and had to be transferred to the intensive care unit to undergo plasma exchange. After three therapeutic plasma exchange cycles, patient 2 recovered consciousness, and both the fever and inflammatory indicators improved. We have reported the specific clinical course of patient in the form of a case report [4]. All patients developed fever after HCART19 cell infusion; nonetheless, in contrast to the MCART19 cell-treated patients, the HCART19 cell-treated patients experienced transient fever that could be controlled by antiallergy and antipyretic drugs. None of the four patients 


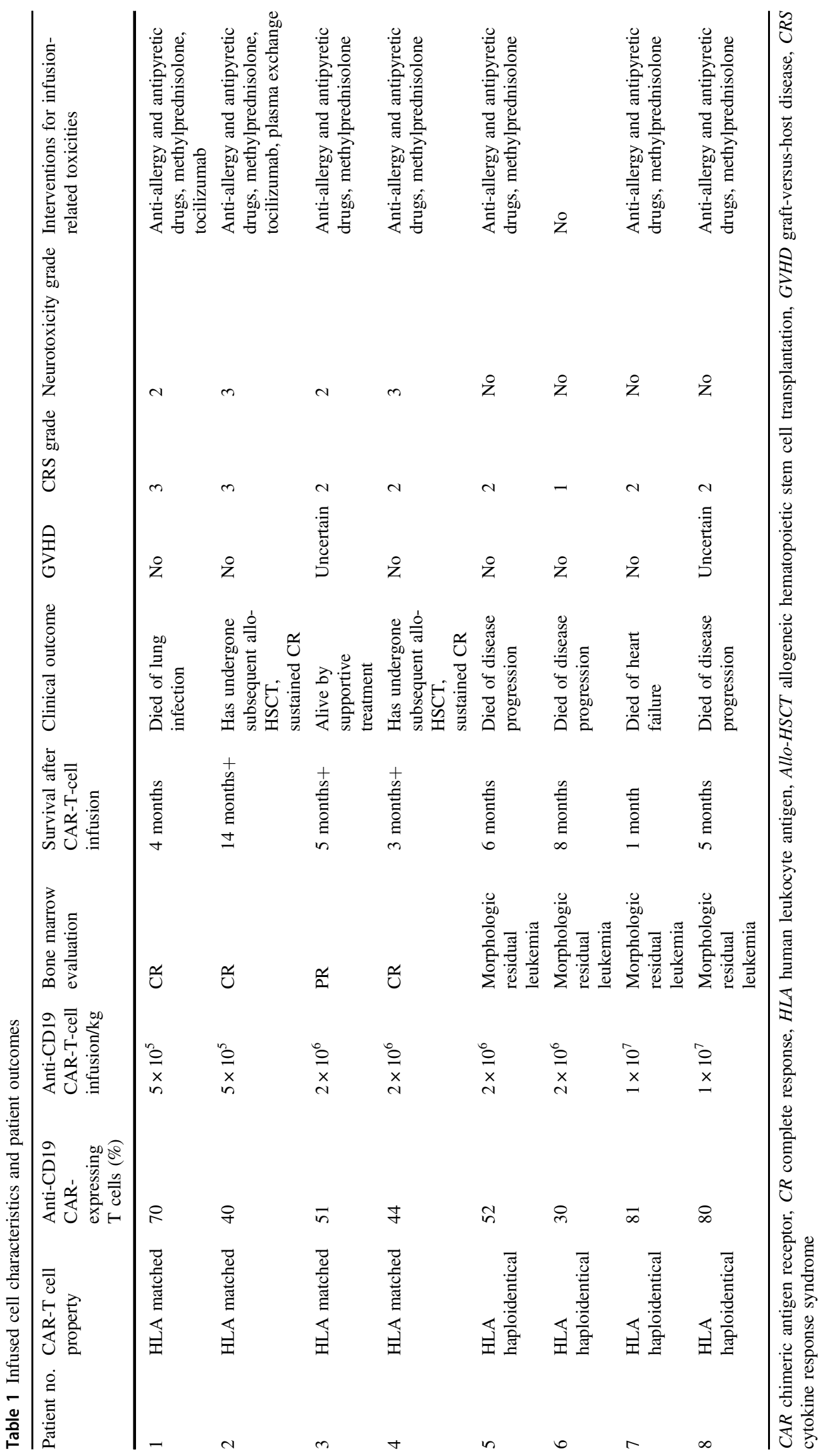


A

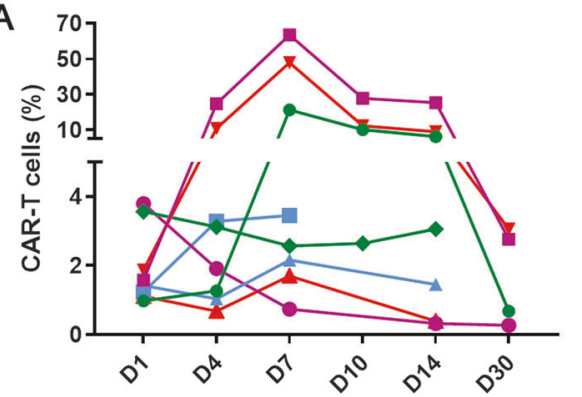

$\rightarrow 1$

$-2$

$\pm 3$

$\rightarrow 4$

$\rightarrow 5$

$-6$

$-7$

$\star 8$
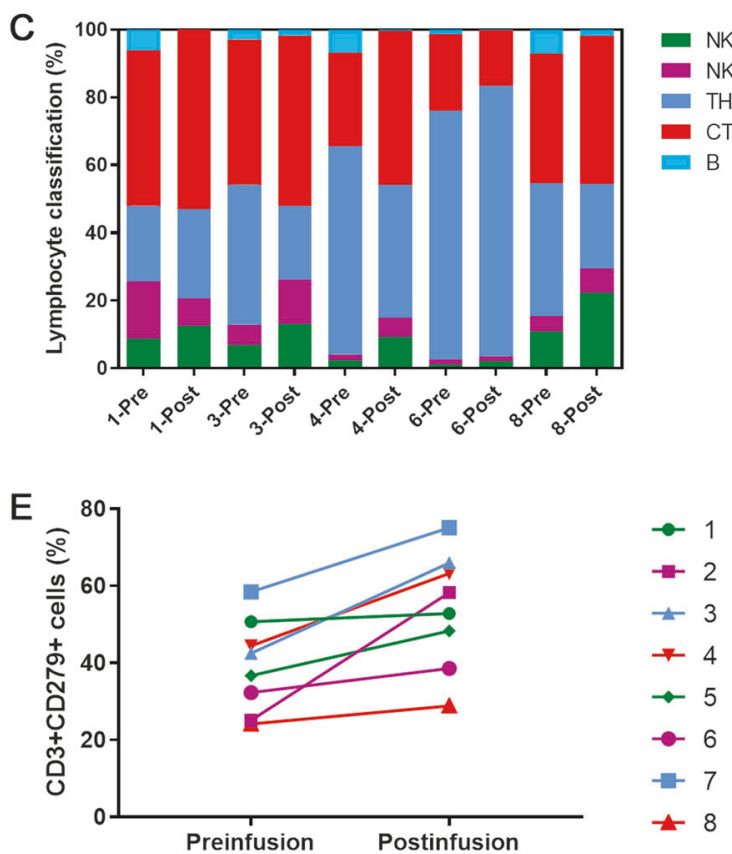

Fig. 1 Allo-CART19 cell expansion, B-cell aplasia and lymphocyte phenotypic analysis occurred in the peripheral blood of patients. a The flow cytometry results showed that the proportion of MCART19 cells increased significantly in the peripheral blood of patients 1,2 , and 4 . Compared with the other five patients, these three patients all had a good response to MCART19 cell therapy. b B-cell dysplasia occurred in patients 1, 2, and 4, who received MCART19 cell therapy. There was only a transient reduction in the proportion of peripheral blood B cells in the four patients treated with HCART19 cell therapy. c NK, NKT, B, TH, and CTL cells evaluated before and after cell infusion by flow cytometry. The number of NK cells in the five patient datasets
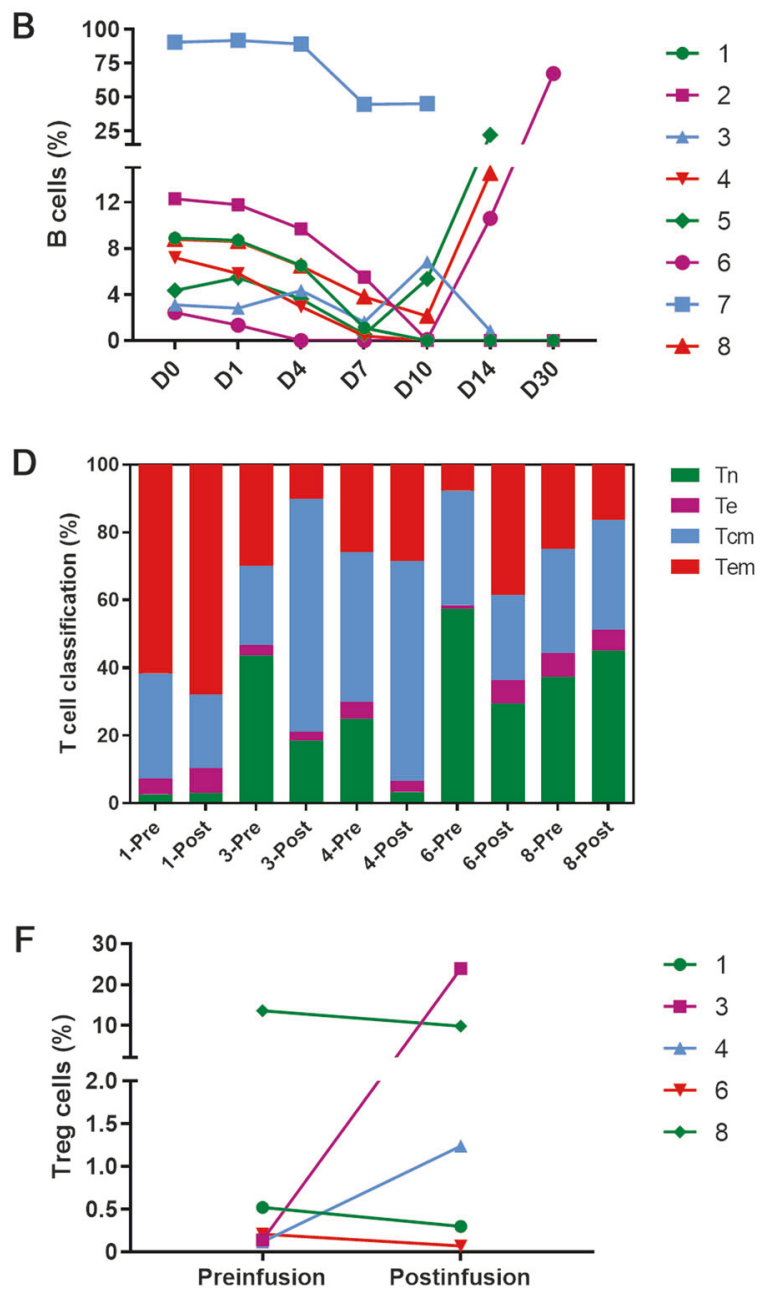

available for review increased after infusion. $\mathbf{d} \mathrm{T}$ cell phenotypic analysis before and after allo-CART19 cell infusion. Data for five patients were available and are displayed. Tn naive $\mathrm{T}$ cell, Te effector $\mathrm{T}$ cell, Tcm central memory $\mathrm{T}$ cell, Tem effector memory $\mathrm{T}$ cell. e Expression of PD-1 on T cells measured by flow cytometry before and after allogeneic CAR-T-cell infusion. All patients completed this evaluation, and almost all patients had increased PD- 1 expression after infusion. f Treg cells measured by flow cytometry before and after allogeneic CAR-T-cell infusion. The proportion of Treg cells in patient 3 increased significantly after treatment

patient 8 again exhibited bloody stool, which was quickly relieved after supplementation with platelets. This adverse event was thought to be caused by the low platelet count rather than GVHD. Patients 3 and 8 experienced transient increases in their total bilirubin levels, and several patients developed elevated ALT, ALP, and GGT levels (Fig. S2AE). Because these patients refused liver biopsy, it is not yet known whether these abnormal indicators were caused by GVHD-related liver damage. Other GVHD evidence, including rash and diarrhea, was not found. One study has reported that allo-CART19 cells within a certain density range have a dose-dependent killing effect on tumor cells but cause platelet count before HCART19 cell infusion. After infusion, 
severe GVHD beyond this range. The absence of GVHD in our cohort may be because the patients were infused with the appropriate dose of allo-CART19 cells. Detailed adverse events are provided in Supplementary Table S2.

Three of the four patients $(75 \%)$ who received the MCART19 cells (patients 1, 2, and 4) achieved complete response (CR) (Table 1). After MCART19 cell infusion, the white blood cell count of patient 1 stayed at a low level. One month after infusion, MCART19 cells became almost undetectable. Possibly due to immunocompromise, the patient developed an infection at 1 month after the infusion and eventually died of the severe infection. Patients 2 and 4 remained in remission for 14 months and 3 months after MCART19 cell therapy and subsequent allo-HSCT, respectively. The two patients received conditioning with total body irradiation, cyclophosphamide, antithymocyte globulin, fludarabine, and cytarabine after 2 months of MCART19 cell infusion, and infused HLA-haploidentical grafts from the younger brother and father, respectively. Two weeks after allo-HSCT, the chimerism monitored by short tandem repeats of these two patients were $96.37 \%$ and $97.51 \%$, respectively, and both achieved complete donor chimerism (100\% of donor alleles) at 1 month after allo-HSCT. Patient 3 was the only patient who underwent MCART19 cell infusion without achieving $\mathrm{CR}$, but a significant reduction in his tumor burden was observed and assessed to be a partial response (PR) by bone marrow biopsies. The four patients treated with the HCART19 cells had a mild response to treatment, as assessed by monitoring the tumor burden in the bone marrow and extramedullary invasion, but relapsed shortly after therapy (Table 1). Patient 6 exhibited subcutaneous tissue infiltration in the right upper limb, and the infiltrating lesion was alleviated after HCART19 cell infusion. Three cases have been reported to have received HCART19 cells before allo-HSCT [5-7]. Two case reports have shown that the use of HCART19 cells as part of a conditioning regimen for alloHSCT has beneficial therapeutic effects [6, 7]. In another case involving the HCART19 cells, no myeloablative conditioning regimen was used, and the patient had a mild response to treatment, which is consistent with the responses we observed [5]. Several reports have found that B-ALL relapses after treatment with autologous CAR-T cells. Even if the antigen recognized by CAR-T cells is still present, the disease will be resistant to a reinfusion of the same CAR-T cells [8-11]. The results for patients 3 and 5 suggest that this resistance cannot be removed even with allo-CART19 cells.

A high percentage of CAR-positive $\mathrm{T}$ cells in the total Tcell population was detected by flow cytometry in the three patients (patients 1, 2, and 4) who achieved CR after MCART19 cell treatment (Fig. 1a). In contrast, the proportion of CAR-positive $\mathrm{T}$ cells did not exceed $5 \%$ in the patients who did not achieve CR. Flow cytometry was performed to monitor the development of B cell aplasia by quantifying the number of CD19-positive B cells, and this methodology can be used as a pharmacodynamic measure of allo-CART19 cell function. For data reliability, we combined specific gene mutations, morphological smears, and CD22 antigen staining to exclude relapse with CD19 antigen loss of malignant B cells. B-cell aplasia occurred in the three patients (patients 1 , 2, and 4) who all achieved CR after MCART19 cell treatment (Fig. 1b). There was only a transient reduction in peripheral blood B cell numbers in the patients who did not achieve CR. These results show that allo-CART19 cell infusion can specifically lyse CD19+ cells in vivo and that the level of CARpositive T-cell expansion is directly related to the therapeutic response (Fig. 1a, b).

The difference in response to the MCART19 cells and HCART19 cells was assessed by analyzing serum cytokine levels, anti-CAR antibody levels, lymphocyte classifications (Table S3), and PD-1 expression on T cells before and after infusion. The results indicated that the NK cell frequencies of five patients increased after allo-CART19 cell infusion (Fig. 1c). The two evaluated patients who received the MCART19 cells and achieved CR had a higher percentage of cells with a Tem phenotype (Tem, CCR7-CD45RO+CD62LCD45RA-) after infusion (Fig. 1d), and this result is similar to that of a previous study [12]. A detailed classification of the T cell phenotypes is provided in the Supplemental Materials (Fig. S3A, B). In all patients, PD-1 expression was higher on endogenous $\mathrm{T}$ cells than on allo-CART19 cells at the time of cell infusion (Fig. 1e). It is worth noting that the only patient who underwent MCART19 cell infusion and did not achieve $\mathrm{CR}$ had a significant increase in the proportion of regulatory $\mathrm{T}$ (Treg) cells and the expression of PD1 on T cells after infusion (Fig. 1e, f). We suspect that these increases are related to the patient's previous infusion of autologous CAR-T cells, but the exact mechanism remains unknown. One study also reported that PD-1 expression is increased after the infusion of allo-CART19 cells derived from posttransplant donors, which corroborates our results [13]. These data show that our alloCART19 cells have properties similar to those of CAR-T cells derived from autologous or posttransplant donors. The lower efficacy of the HCART19 cells compared with that of the MCART19 cells may be due to the heterogeneity of the former in inducing graft rejection. It is unclear whether strengthening immunosuppressive treatment prior to infusion may enhance the anti-tumor effect of HCART19 cells, but this may also increase the risk of GVHD. However, this is only a hypothesis, and it still needs more evidence to be established.

Together, these cases suggest that allogeneic CAR-T cell therapy is feasible in R/R B-ALL and overcome limitation of autologous CAR-T cells, thus may be one possible regimen before the era of off-the-shelf "universal" CAR-T cell therapy. Compared with HCART19 cell infusion, MCART19 cell infusion had a higher CR rate and was 
accompanied by more severe toxic side effects. Although we did not observe GVHD or uncontrolled infusion-related toxicity, allo-CART19 cell therapy still requires close attention to GVHD and toxicity events. We have described only eight cases in a single center, and clinical trial studies to expand the sample size are urgently needed.

Acknowledgements This work was supported by grants from the National Natural Sciences Foundation of China (81970180; to MZ), the National Natural Sciences Foundation of China (81800105; to WL), the Tianjin Key Natural Science Foundation (17JCZDJC35800 to MZ), and the Tianjin Key Science and Technology Program (16KG110 to MZ), as well as Tianjin First Central Hospital.

Author contributions MFZ designed the research; XJ, YQC, LQW, XX, YLJ, QL, HZ, YYJ, and JXM performed the research; XJ, YQC, LQW, RS, LC, XYH, WYL, and CCL analyzed the data; XJ, YQC, and LQW wrote the manuscript; and XJ, YQC, LQW, and MFZ revised the manuscript. All authors approved the final version of the manuscript.

\section{Compliance with ethical standards}

Conflict of interest The authors declare that they have no conflict of interest.

Ethics approval and consent to participate This study was conducted according to the principles of the Declaration of Helsinki and with the approval of the Ethics Committee of Tianjin First Central Hospital. All the enrolled patients or their families provided written informed consent.

Publisher's note Springer Nature remains neutral with regard to jurisdictional claims in published maps and institutional affiliations.

Open Access This article is licensed under a Creative Commons Attribution 4.0 International License, which permits use, sharing, adaptation, distribution and reproduction in any medium or format, as long as you give appropriate credit to the original author(s) and the source, provide a link to the Creative Commons license, and indicate if changes were made. The images or other third party material in this article are included in the article's Creative Commons license, unless indicated otherwise in a credit line to the material. If material is not included in the article's Creative Commons license and your intended use is not permitted by statutory regulation or exceeds the permitted use, you will need to obtain permission directly from the copyright holder. To view a copy of this license, visit http://creativecommons. org/licenses/by/4.0/.

\section{References}

1. June $\mathrm{CH}$, Sadelain M. Chimeric antigen receptor therapy. New Engl J Med. 2018;379:64-73.

2. Torikai H, Cooper LJ. Translational implications for off-the-shelf immune cells expressing chimeric antigen receptors. Mol Ther. 2016;24:1178-86.

3. Kebriaei P, Singh H, Huls MH, Figliola MJ, Bassett R, Olivares S, et al. Phase I trials using sleeping beauty to generate CD19specific CAR T cells. J Clin Invest. 2016;126:3363-76.

4. Xiao X, He X, Li Q, Zhang H, Meng J, Jiang Y, et al. Plasma exchange can be an alternative therapeutic modality for severe cytokine release syndrome after chimeric antigen receptor- $\mathrm{T}$ cell infusion: a case report. Clin Cancer Res. 2019;25:29-34.

5. Zhang JP, Zhang R, Tsao ST, Liu YC, Chen X, Lu DP, et al. Sequential allogeneic and autologous CAR-T-cell therapy to treat an immune-compromised leukemic patient. Blood Adv. 2018;2: $1691-5$.

6. Cai B, Guo M, Wang Y, Zhang Y, Yang J, Guo Y, et al. Coinfusion of haplo-identical CD19-chimeric antigen receptor T cells and stem cells achieved full donor engraftment in refractory acute lymphoblastic leukemia. J Hematol Oncol. 2016;9:131.

7. Zhang C, Kong PY, Li SQ, Chen T, Ni X, Li YY, et al. Donorderived CAR-T cells serve as a reduced-intensity conditioning regimen for haploidentical stem cell transplantation in treatment of relapsed/refractory acute lymphoblastic leukemia: case report and review of the literature. J Immunother. 2018;41: 306-11.

8. Sadelain M, Riviere I, Riddell S. Therapeutic T cell engineering. Nature. 2017;545:423-31.

9. Fry TJ, Shah NN, Orentas RJ, Stetler-Stevenson M, Yuan CM, Ramakrishna S, et al. CD22-targeted CAR T cells induce remission in B-ALL that is naive or resistant to CD19-targeted CAR immunotherapy. Nat Med. 2018;24:20-8.

10. Sotillo E, Barrett DM, Black KL, Bagashev A, Oldridge D, Wu G, et al. Convergence of acquired mutations and alternative splicing of CD19 enables resistance to CART-19 immunotherapy. Cancer Disco. 2015;5:1282-95.

11. Majzner RG, Mackall CL. Tumor antigen escape from CAR T-cell therapy. Cancer Disco. 2018;8:1219-26.

12. Kochenderfer JN, Dudley ME, Carpenter RO, Kassim SH, Rose JJ, Telford WG, et al. Donor-derived CD19-targeted T cells cause regression of malignancy persisting after allogeneic hematopoietic stem cell transplantation. Blood. 2013;122: 4129-39.

13. Brudno JN, Somerville RP, Shi V, Rose JJ, Halverson DC, Fowler $\mathrm{DH}$, et al. Allogeneic $\mathrm{T}$ cells that express an anti-cd19 chimeric antigen receptor induce remissions of b-cell malignancies that progress after allogeneic hematopoietic stem-cell transplantation without causing graft-versus-host disease. J Clin Oncol. 2016;34: $1112-21$. 